\title{
The effect of adsorbates on the electrical stability of graphene studied by transient photocurrent spectroscopy
}

\author{
S. B. Kalkan, ${ }^{1}$ H. Aydın, ${ }^{1,2}$ D. Özkendir, ${ }^{1,2}$ and C. Çelebi ${ }^{1, a)}$ \\ ${ }^{1}$ Quantum Device Laboratory, Department of Physics, Izmir Institute of Technology, Izmir 35430, Turkey \\ ${ }^{2}$ Department of Material Science and Engineering, İzmir Institute of Technology, Izmir 35430, Turkey
}

(Received 1 November 2017; accepted 18 December 2017; published online 3 January 2018)

\begin{abstract}
Adsorbate induced variations in the electrical conductivity of graphene layers with two different types of charge carriers are investigated by using the Transient Photocurrent Spectroscopy (TPS) measurement technique. In-vacuum TPS measurements taken for a duration of $5 \mathrm{ks}$ revealed that the adsorption/desorption of atmospheric adsorbates leads to more than a $110 \%$ increment and a $45 \%$ decrement in the conductivity of epitaxial graphene (n-type) and chemical vapor deposition graphene (p-type) layers on semi-insulating silicon carbide ( $\mathrm{SiC}$ ) substrates, respectively. The graphene layers on $\mathrm{SiC}$ are encapsulated and passivated with a thin $\mathrm{SiO}_{2}$ film grown by the Pulsed Electron Deposition method. The measurements conducted for short periods and a few cycles showed that the encapsulation process completely suppresses the time dependent conductivity instability of graphene independent of its charge carrier type. The obtained results are used to construct an experimental model for identifying adsorbate related conductivity variations in graphene and also in other 2D materials with an inherently high surface-to-volume ratio. Published by AIP Publishing. https://doi.org/10.1063/1.5011454
\end{abstract}

Two-dimensional (2D) materials such as graphene, $\mathrm{MoS}_{2}$, and BN exhibit exciting properties as they are thinned down from their bulk counterparts to their physical limits. Owing to their superior surface-to-volume ratio and hence their sensitivity to extrinsic adsorbates, graphene, and other 2D materials are considered to be adapted for gas and humidity sensing technology. ${ }^{1-3}$ However, the unique property of these $2 \mathrm{D}$ structures presents serious problems when they are desired to be used as electrically stable components in electronic or optoelectronic devices operating under atmospheric conditions. The adsorbates like $\mathrm{O}_{2}$ and $\mathrm{H}_{2} \mathrm{O}$ in air serve as surface trap states, and hence, the adsorption/desorption of these molecules drastically alters the electronic transport characteristics of 2D materials ${ }^{4-6}$ via the charge transfer doping effect. For example, it has been shown that atmospheric adsorbates degrade the conductivity of monolayer $\mathrm{MoS}_{2}$ by more than an order of magnitude. ${ }^{7}$

Graphene forms a Schottky junction when it is transferred onto the surface of technologically important conventional semiconductors like $\mathrm{Si}, \mathrm{GaAs}$, and $\mathrm{SiC}^{8-10}$ because of the fact that the graphene/semiconductor heterojunction is expected to exhibit a strong rectification which can be utilized for Schottky barrier devices like tunneling field effect transistors and photodetectors with relatively low leakage currents. ${ }^{11,12}$ However, adsorbate induced alteration in graphene's conductivity causes undesirable variations ${ }^{4,13}$ in the effective Schottky barrier height ${ }^{14}$ and consequently gives rise to instabilities in device performance. Therefore, in order to maintain long term electrical stability of the devices, the interaction between the 2D materials and surrounding gas molecules should be necessarily prevented.

In this letter, we report an experimental model to reveal the effect of adsorption/desorption of atmospheric adsorbates

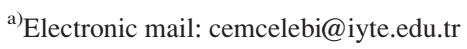

on the electrical characteristics of both n-type and p-type graphene layers which were either transferred or directly grown on semi-insulating (SI) $\mathrm{SiC}$ substrates. In-vacuum transient photocurrent spectroscopy (TPS) measurements taken under ultraviolet (UV) light illumination showed that the adsorbates modify the electrical conductivity of graphene depending only on its charge carrier type and hinder the formation of photogenerated charge carriers in the depletion region of $\mathrm{SiC}$ underneath. For comparison, a set of graphene/ $\mathrm{SiC}$ samples with two different carrier types in the graphene layer were encapsulated with the $\mathrm{SiO}_{2}$ thin film by using the state-of-the-art Pulsed Electron Deposition (PED) technique. The TPS measurements revealed that the photoresponse time of the samples with a bare graphene layer was greatly improved (independent of graphene's carrier type) from hours to a second when their surfaces are passivated with a PED grown $\mathrm{SiO}_{2}$ encapsulation layer. The experimentally obtained results are used to resolve the mechanism behind the adsorbate induced variations in the charge carrier dynamics of graphene and also other 2D materials.

It is known from the literature that graphene layers grown by chemical vapor deposition (CVDG) has strong p-type carrier density, ${ }^{15,16}$ while the epitaxial graphene (EG) layers produced on the $\mathrm{Si}$-face surface of the $\mathrm{SiC}$ substrate exhibits an inherent n-type conductivity. ${ }^{17-19}$ Therefore, in order to make a viable comparison between two different charge carrier types but for the same material, we used both monolayer CVDG and EG that were either transferred or directly grown on SI-SiC substrates. The details of the growth procedures for CVDG and EG can be found in Refs. 20 and 21, respectively. The presence of graphene and its thickness were determined by single point Raman spectroscopy measurements. The Raman spectra of CVDG on copper $(\mathrm{Cu})$ foil and $\mathrm{EG}$ on the $\mathrm{SiC}$ substrate were acquired right after each growth procedure. As shown in Fig. 1(a), the 

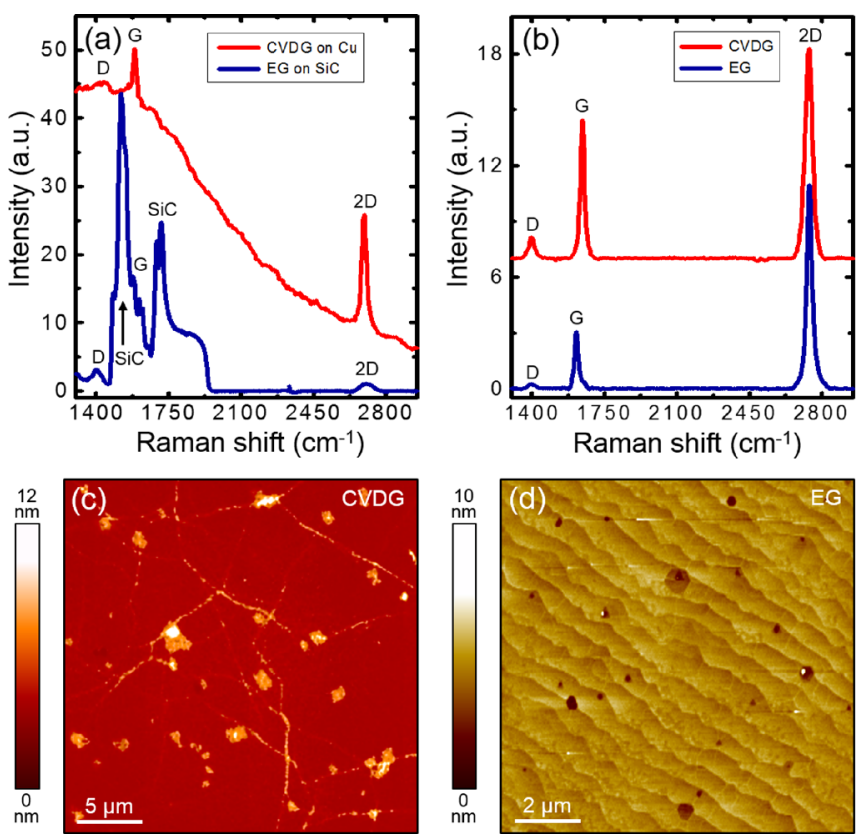

FIG. 1. The Raman spectra of CVDG on Cu and EG on SiC (a) without and (b) with background attenuation. AFM topography images of (c) CVDG on $\mathrm{Cu}$ and (d) EG on $\mathrm{SiC}$ substrates.

Raman spectrum of CVDG retains a curve like $\mathrm{Cu}$ induced florescence background, while the Raman spectrum of EG contains $\mathrm{SiC}$ associated band peaks. Graphene related $\mathrm{D}, \mathrm{G}$, and $2 \mathrm{D}$ peaks were resolved in all the measurements. Figure 1(b) displays the background subtracted Raman spectra of CVDG and EG. ${ }^{22}$ The obtained Raman profiles reveal the characteristics $G$ and single Lorentzian 2D peaks that are similar to those of mechanically exfoliated single layer graphene on a $300 \mathrm{~nm}$ thick $\mathrm{SiO}_{2}$ substrate. ${ }^{23}$ The strong $\mathrm{G}$ peak and the weak D peak indicate good graphitic quality, and the large $2 \mathrm{D}$ to $\mathrm{G}$ peak intensity ratios $\left(\mathrm{I}_{2 \mathrm{D}} / \mathrm{I}_{\mathrm{G}}>2\right)$ confirm the monolayer nature of both CVDG and EG. ${ }^{24}$ The morphology of the grown graphene layers was determined by tapping mode Atomic Force Microscopy (AFM) topography measurements. Figures 1(c) and 1(d) show the AFM images of transferred CVDG and EG on the SiC substrate, respectively. As seen in Fig. 1(c), the CVDG layer contains wrinkles and residues originating from the growth procedures and photoresist assisted transfer of CVDG from $\mathrm{Cu}$ foil onto the $\mathrm{SiC}$ substrate. The AFM image of EG exhibits typical SiC background based large terraces ${ }^{21}$ and graphene related flaky structures appearing with blurry wrinkles lying on these terraces [Fig. 1(d)]. The small amount of local but darker regions corresponds to a few layer EG flakes.

Following the Raman and AFM analysis, $\mathrm{Cr} / \mathrm{Au}(3 \mathrm{~nm} /$ $80 \mathrm{~nm}$ ) interconnect source/drain pads were thermally deposited on the sides of graphene layers for conducting twoterminal I-V measurements. The surfaces of a set of CVDG and EG samples, comprising source/drain electrodes, were encapsulated with a $100 \mathrm{~nm}$ thick $\mathrm{SiO}_{2}$ layer by the PED method. The TPS measurements of the samples were done under $254 \mathrm{~nm}$ wavelength UV light illumination inside a high vacuum chamber with a base pressure of about $5 \times 10^{-5}$ mbar. For the TPS experiments, an electronic shutter mechanism is coupled to the UV light source and the photocurrent data of the samples were acquired at a bias voltage of $\mathrm{V}_{\mathrm{b}}=2 \mathrm{~V}$. Prior to the TPS measurements, we conducted I-V measurements for both CVDG and EG before and after the $\mathrm{SiO}_{2}$ encapsulation process, and the obtained results are compared in Figs. 2(a) and 2(b). The initial resistance of bare CVDG (B-CVDG) was measured to be $1.1 \mathrm{k} \Omega$, and after the encapsulation, its resistance was found to be increased to 4.7 $\mathrm{M} \Omega$. The initial resistance of bare $\mathrm{EG}(\mathrm{B}-\mathrm{EG})$ is $6.5 \mathrm{k} \Omega$, while the encapsulated EG (E-EG) has the resistance of 1.2 $\mathrm{M} \Omega$. The increment in the resistance of the samples was attributed to the passivation of the dangling bonds responsible for the charge transport in the graphene layer.

Although there are a variety of studies regarding the adsorption/desorption of $\mathrm{O}_{2}$ and $\mathrm{H}_{2} \mathrm{O}$ molecules by graphene, ${ }^{4,13}$ there are no TPS measurements conducted to obtain a deep insight into the adsorption/desorption induced variations in the conductivity of graphene layers with two different charge carrier types. To promote desorption of adsorbates which were already stuck on graphene in air, the samples were exposed to UV light for a period of $5 \mathrm{ks}$ under high vacuum conditions. The irradiation wavelength is specifically selected to be $254 \mathrm{~nm}$ since it is energetically sufficient enough to desorb $\mathrm{O}_{2}$ and $\mathrm{H}_{2} \mathrm{O}$ molecules from the graphene layer $^{13,25}$ and at the same time to generate excitons in the depletion region of the underlying $\mathrm{SiC}$ crystal due to its wide bandgap energy $\left(\mathrm{E}_{\mathrm{g}}=3.2 \mathrm{eV}\right)$.

Figure 2(c) shows that as the UV light desorbs the adsorbates, the current of B-CVDG decreases monotonously in contrast to the increase in the current for B-EG. This opposite behavior seen in the current characteristics suggests that the charge carrier types in these two graphene layers are indeed different from each other. After $5 \mathrm{ks}$ period of UV light illumination, the changes in the currents of B-CVDG and B-EG were measured as high as $45 \%$ and $110 \%$, respectively. When the UV light is turned off, the adsorbates with lower partial pressure in the middle $10^{-5}$ mbar vacuum range
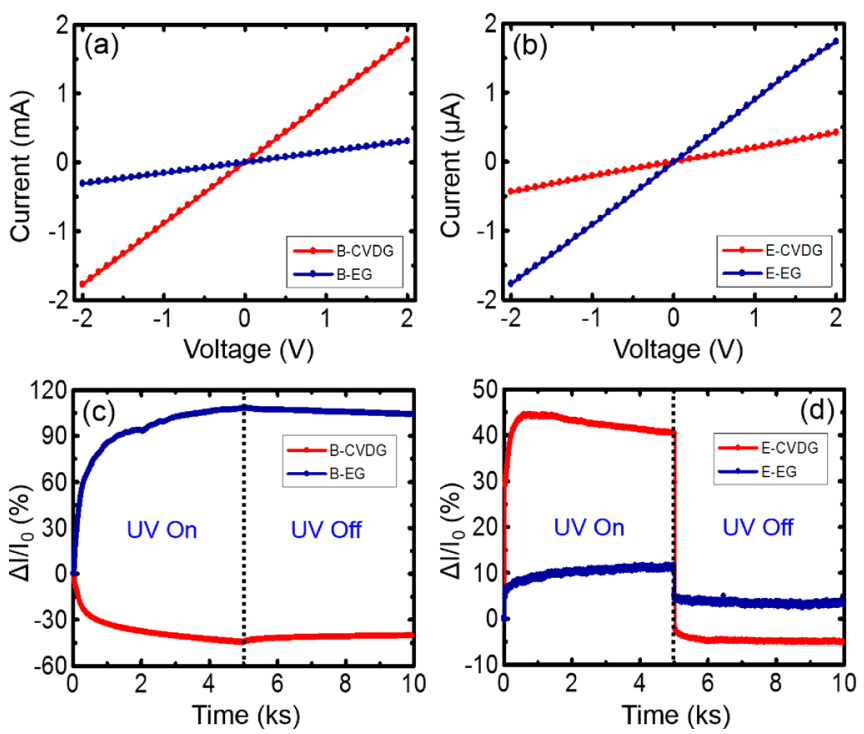

FIG. 2. The I-V characteristics of CVDG and EG layers on SI-SiC (a) before and (b) after the $\mathrm{SiO}_{2}$ encapsulation process. The change in the currents of (c) CVDG and (d) EG samples that were exposed to $254 \mathrm{~nm}$ UV light for a period of $5 \mathrm{ks}$ under high vacuum conditions. After a period of $5 \mathrm{ks}$, the UV light is turned off and the samples are let to relax in vacuum for another $5 \mathrm{ks}$. 
tend to stick back onto the graphene layer and hence give rise to a slight variation in the final current level of the samples. We have found that even after a period of $5 \mathrm{ks}$, the current of the sample could not reach its initial value measured before the UV light illumination. However, unlike the B-CVDG and B-EG, the UV light leads to a rapid increase in the measured currents of the $\mathrm{SiO}_{2}$ encapsulated ones [Fig. 2(d)]. When the UV light is turned off, the current drops sharply down to its initial state within only one second. Such a fast on/off transition in the currents of encapsulated samples is dominated by the photogenerated charge carriers created in the depletion region of the $\mathrm{SiC}$ substrate underneath and not by adsorption/desorption of the adsorbates on the graphene layer.

The TPS measurements applied for short periods and a couple of cycles provide insight into the distinct behaviors behind the above mentioned current characteristics of the samples. For the TPS measurements, we set the shutter on/ off period to $30 \mathrm{~s}$ for three cycles. Prior to the experiments, all the samples were exposed to UV light in vacuum for a duration of $10 \mathrm{ks}$ to make sure that the adsorbates are removed in a large extent from the graphene layer. The results of TPS measurements acquired before and after the encapsulation process are shown in Fig. 3. The spectra that belong to B-CVDG and E-CVDG are compared in Figs. 3(a) and 3(b), respectively. When the shutter is closed, a sudden drop in the current is observed within a second for B-CVDG [Fig. 3(a)]. Then, in a couple of seconds, a trace amount of adsorbates with lower partial pressure in vacuum (middle $10^{-5}$ mbar) is adsorbed back by the graphene layer over time, resulting in an exponential growth of the current. Under the UV light illumination, a sharp increment in the current occurred also within a second, which is followed by a rapid exponential decay. From the literature, we are aware of the fact that the adsorption of $\mathrm{O}_{2}$ and/or $\mathrm{H}_{2} \mathrm{O}$ molecules dopes graphene with holes. ${ }^{4,13}$ This explains well the time dependent but slow variation of the current in our B-CVDG when the UV light is either turned on or off. Since the adsorption rate with the measured time constant of $\tau_{\mathrm{ad}}=21.7 \mathrm{~s}$ is smaller than the desorption rate $\left(\tau_{\mathrm{des}}=13.2 \mathrm{~s}\right)$, an upward trend was observed in the overall current variation within a couple of on/off cycles. Unlike B-CVDG, the TPS plot of the encapsulated one exhibits uniform and stable profiles, revealing that the sample had an excellent photocurrent reversibility and a fast response speed [Fig. 3(b)]. In addition, the magnitude of the current change in CVDG samples was found to be increase from $1 \%$ to $34 \%$ when it is encapsulated with the $\mathrm{SiO}_{2}$ layer.
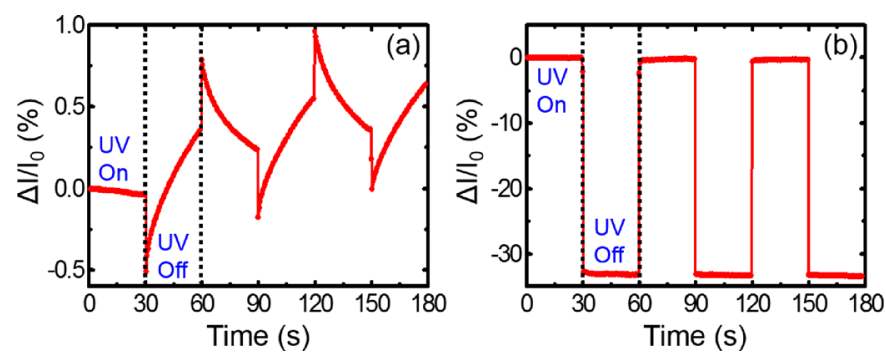

For B-EG, a relatively weak but fast decrement in the current is followed by an exponential decay when the shutter is closed [Fig. 3(c)]. Similar to that observed for B-CVDG, we measured a sudden rise in the current within a second after the shutter is opened. However, following the first steep rise, the current increased exponentially as a function of time in contrast to the exponential decay of the current measured for B-CVDG. This observed behavior in the current variation for B-EG cannot be explained in terms of adsorbate induced hole doping process. The measured TPS characteristics of our B-EG are similar to those of n-type metal-oxide semiconductors like $\mathrm{ZnO}$ thin films. ${ }^{26-28}$ The adsorbates that were stuck on the surface of the n-type $\mathrm{ZnO}$ film trap the free electrons and thus lead to a drastic drop in the current under atmospheric conditions. Analogously, as the UV light desorbs the adsorbates from the B-EG layer, the trapped electrons are released over time and therefore give rise to an exponential growth in the current. For B-EG, the adsorption rate with the measured time constant of $\tau_{\mathrm{ad}}=27.5 \mathrm{~s}$ is also smaller than the desorption rate $\left(\tau_{\mathrm{des}}=17.9 \mathrm{~s}\right)$. However, unlike B-CVDG, the difference between the adsorption rate and the desorption rate for B-EG causes a downward trend in the overall current variation within a couple of on/off cycles. As displayed in Fig. $3(d)$, the current of the sample could be reversibly modulated by UV illumination, indicating good reproducibility and stability of E-EG. The small amplitude variations seen in the measured current are attributed to the hole traps present at the EG/SiC interface. ${ }^{19}$ The photoresponsivities of encapsulated graphene layers are compared with each other for a UV irradiation output power of $3 \mathrm{~mW}$. The photoresponsivity of ECVDG is measured to be $90 \mu \mathrm{A} / \mathrm{W}$, which is much larger than the one measured for E-EG $(32 \mu \mathrm{A} / \mathrm{W})$.

The illustrations in Fig. 4 display the adsorption/desorption induced modifications in the charge carrier dynamics of CVDG and EG samples and provide an insight into the experimental results presented in Fig. 3. For the samples with a bare graphene layer, the conductivity is modified by the adsorption/ desorption of adsorbates and photogenerated charge carriers from the underlying $\mathrm{SiC}$ substrate together. The experimentally obtained results manifest that the conductivity of BCVDG $\left(\sigma_{C V D G}\right)$ changes in the form of $\Delta \sigma_{C V D G}(t) \propto\left[\sigma_{p h}\right.$ $-\sigma_{a d}(t)$ ] [compare Figs. 3(a) and 4(a)], whereas the change in the conductivity of B-EG $(\sigma)$ takes the form of $\Delta \sigma_{E G}(t) \propto$ $\left[\sigma_{p h}+\sigma_{a d}(t)\right]$ [compare Figs. 3(c) and 4(c)]. Here, $\sigma_{p h}$ is the contribution of photogenerated charge carriers and $\sigma_{a d}(t)$ is the contribution of adsorbates to graphene's conductivity. Compared to B-EG, the contribution of these photogenerated charge carriers to the overall conductivity is more pronounced
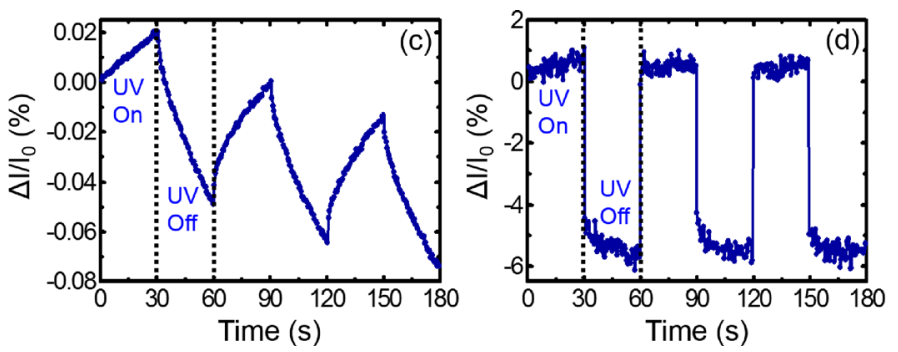

FIG. 3. Short-period TPS measurements of CVDG (a) before and (b) after the $\mathrm{SiO}_{2}$ encapsulation process. Short-period TPS measurements of EG (c) before and (d) after the $\mathrm{SiO}_{2}$ encapsulation process. Rectified and stable TPS characteristics were achieved for both CVDG and EG after the SiO 2 encapsulation. 
(a)

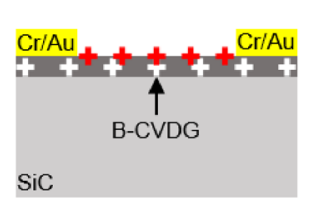

Before

Illumination

(c)

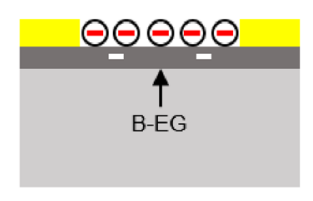

Before

Illumination

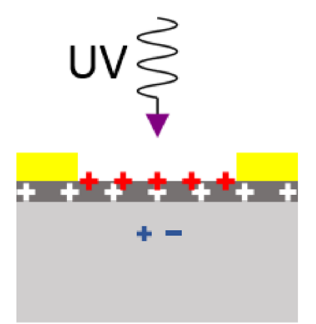

After $1 \mathrm{~s}$

Illumination

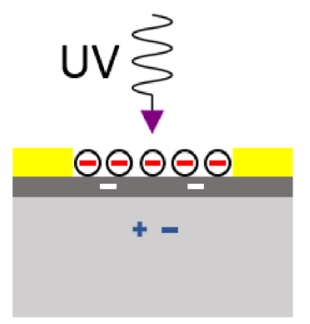

After $1 \mathrm{~s}$

Illumination

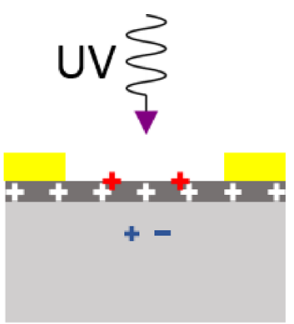

After $30 \mathrm{~s}$

Illumination

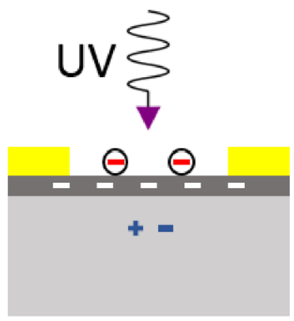

After $30 \mathrm{~s}$

Illumination

(b)

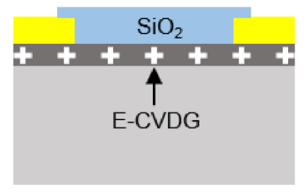

Before

Illumination

(d)

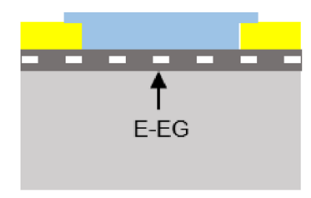

Before

Illumination

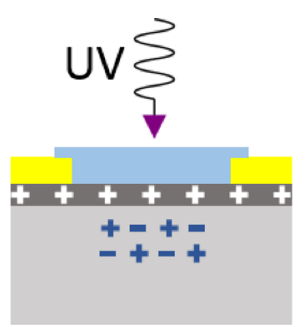

After

Illumination

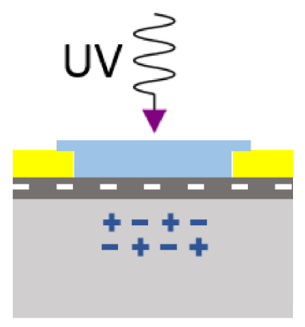

After

Illumination

FIG. 4. The schematic side view of adsorption/desorption driven charge carrier dynamics for (a) B-CVDG, (b) E-CVDG, (c) B-EG, and (d) E-EG. White colored charges represent the intrinsic charge carriers in graphene layers, red colored charges depict the injected (for CVDG) or trapped (for EG) charge carriers by the adsorbates, and the blue colored charges are the photogenerated charge carriers formed in the depletion region of the SiC substrate beneath graphene layers (the sketch is not scaled).

in the B-CVDG sample, because of the fact that unlike B-EG, we observed a spike-like sudden rise in the current of BCVDG when the UV light is either turned on or off. As can be understood from the TPS measurements shown in Figs. 3(a) and $3(\mathrm{c}), \sigma_{a d}(t)$ is indeed a time dependent exponentially varying function. The adsorbate layer on graphene absorbs most of the UV photons impinging on the sample surface for the desorption process and thus strongly hinders the formation of photogenerated charge carriers in the depletion layer of $\mathrm{SiC}$ underneath. When the UV light is off, the change in the conductivity becomes $\Delta \sigma_{C V D G}(t) \propto\left[\sigma_{a d}(t)-\sigma_{p h}\right]$ for B-CVDG and $\Delta \sigma_{E G}(t) \propto\left[-\left(\sigma_{p h}+\sigma_{a d}(t)\right)\right]$ for B-EG due to the adsorption of the adsorbates with low partial pressure in the corresponding vacuum level. However, when the samples are encapsulated, the contribution of adsorbates to the graphene's conductivity is suppressed, and therefore, the UV light produces only the photogenerated carriers in the depletion layer of $\mathrm{SiC}$. These photogenerated charge carriers modify the conductivity of the graphene layers via the charge transfer doping process, because of the fact that the conductivity variation in both the encapsulated samples takes a time independent form of $\Delta \sigma \propto \sigma_{p h}$ as schematically depicted in Figs. 4(b) and 4(d).

In summary, we have investigated the impact of atmospheric adsorbates on the electrical stability of graphene layers with two different charge carrier types by using the Transient Photocurrent Spectroscopy technique. The measurements proved that the adsorption/desorption of adsorbates indeed modifies the conductivity of graphene, but in a distinct manner, depending on whether it is n-type (as in epitaxial graphene) or p-type doped (as in CVD graphene), and acts as the main source for time dependent electrical instability. Adsorbate induced variations in the electrical conductivity of n-type graphene were found to be consistent with the conductivity instabilities in n-type metal-oxide semiconductors like
$\mathrm{ZnO}$ thin films subject to the atmospheric environment. For $\mathrm{p}-$ type materials with a high surface-to-volume ratio, we also expect adsorbate induced conductivity instabilities, but in the same manner as we experimentally observed for CVD graphene in this study. The encapsulation of graphene with PED grown $\mathrm{SiO}_{2}$ was found to block the adsorption/desorption of atmospheric adsorbates and therefore maintains the electrical stability of graphene independent of its charge carrier type. Our experimentally obtained results can be used to interpret the adsorbate induced conductivity variations that may occur in other materials like 2D systems with an inherently high surface-to-volume ratio.

The authors would like to thank Serap Yiğen for Pulsed Electron Deposition experiments, Damla Yeşilpınar for her help in the sample preparation procedures, and Sparks Electronics Ltd., Turkey, for providing us with the stainless steel hard masks that were used in the sample fabrication processes.

${ }^{1}$ L. Kong, A. Enders, T. S. Rahman, and P. A. Dowben, J. Phys. Condens. Matter 26, 443001 (2014).

${ }^{2}$ F. Schedin, A. K. Geim, S. V. Morozov, E. W. Hill, P. Blake, M. I. Katsnelson, and K. S. Novoselov, Nat. Mater. 6, 652 (2007).

${ }^{3}$ Y.-H. Zhang, Y.-B. Chen, K.-G. Zhou, C.-H. Liu, J. Zeng, H.-L. Zhang, and Y. Peng, Nanotechnology 20, 185504 (2009).

${ }^{4}$ Y. Yang and R. Murali, Appl. Phys. Lett. 98, 93116 (2011).

${ }^{5}$ A. G. Shiravizadeh, R. Yousefi, S. M. Elahi, and S. A. Sebt, Phys. Chem. Chem. Phys. 19, 18089 (2017).

${ }^{6}$ R. Yousefi, H. R. Azimi, M. R. Mahmoudian, and W. J. Basirun, Appl. Surf. Sci. 435, 886 (2018).

${ }^{7}$ D. Jariwala, V. K. Sangwan, D. J. Late, J. E. Johns, V. P. Dravid, T. J. Marks, L. J. Lauhon, and M. C. Hersam, Appl. Phys. Lett. 102, 173107 (2013).

${ }^{8}$ S. Tongay, T. Schumann, and A. F. Hebard, Appl. Phys. Lett. 95, 222103 (2009).

${ }^{9}$ C. C. Chen, M. Aykol, C. C. Chang, A. F. J. Levi, and S. B. Cronin, Nano Lett. 11, 1863 (2011). 
${ }^{10}$ S. Tongay, M. Lemaitre, T. Schumann, K. Berke, B. R. Appleton, B. Gila, and A. F. Hebard, Appl. Phys. Lett. 99, 102102 (2011).

${ }^{11}$ Y. An, A. Behnam, E. Pop, and A. Ural, Appl. Phys. Lett. 102, 13110 (2013).

${ }^{12}$ X. Li, M. Zhu, M. Du, Z. Lv, L. Zhang, Y. Li, Y. Yang, T. Yang, X. Li, K. Wang, H. Zhu, and Y. Fang, Small 12, 595 (2016).

${ }^{13}$ S. Ryu, L. Liu, S. Berciaud, Y. J. Yu, H. Liu, P. Kim, G. W. Flynn, and L. E. Brus, Nano Lett. 10, 4944 (2010).

${ }^{14}$ M. W. K. Nomani, A. Singh, V. Shields, M. Spencer, G. Tompa, N. Sbrockey, and G. Koley, in IEEE Conference on Nanotechnology (2011), Vol. 2, p. 1317.

${ }^{15}$ J. W. Suk, W. H. Lee, J. Lee, H. Chou, R. D. Piner, Y. Hao, D. Akinwande, and R. S. Ruoff, Nano Lett. 13, 1462 (2013).

${ }^{16}$ A. Pirkle, J. Chan, A. Venugopal, D. Hinojos, C. W. Magnuson, S. McDonnell, L. Colombo, E. M. Vogel, R. S. Ruoff, and R. M. Wallace, Appl. Phys. Lett. 99, 122108 (2011).

${ }^{17}$ S. Kopylov, A. Tzalenchuk, S. Kubatkin, and V. I. Fal'Ko, Appl. Phys. Lett. 97, 112109 (2010).

${ }^{18}$ J. Eriksson, R. Pearce, T. Iakimov, C. Virojanadara, D. Gogova, M. Andersson, M. Syväjärvi, A. Lloyd Spetz, and R. Yakimova, Appl. Phys. Lett. 100, 241607 (2012).
${ }^{19}$ A. N. Sidorov, K. Gaskill, M. Buongiorno Nardelli, J. L. Tedesco, R. L. Myers-Ward, C. R. Eddy, T. Jayasekera, K. W. Kim, R. Jayasingha, A. Sherehiy, R. Stallard, and G. U. Sumanasekera, J. Appl. Phys. 111, 113706 (2012).

${ }^{20}$ H. Aydin, C. Bacaksiz, N. Yagmurcukardes, C. Karakaya, O. Mermer, M. Can, R. T. Senger, H. Sahin, and Y. Selamet, Appl. Surf. Sci. 428, 1010 (2018).

${ }^{21}$ E. Kuşdemir, D. Özkendir, V. Fırat, and C. Çelebi, J. Phys. D:. Appl. Phys. 48, 95104 (2015).

${ }^{22}$ S. Shivaraman, M. V. S. Chandrashekhar, J. J. Boeckl, and M. G. Spencer, J. Electron. Mater. 38, 725 (2009).

${ }^{23}$ D. Graf, F. Molitor, K. Ensslin, and C. Stampfer, Nano Lett. 7, 238 (2007).

${ }^{24}$ Z. Lin, X. Ye, J. Han, Q. Chen, P. Fan, H. Zhang, D. Xie, H. Zhu, and M. Zhong, Sci. Rep. 5, 11662 (2015).

${ }^{25}$ E. Blakely and S. Antonio, U.S. patent 4,660,297 (April 28, 1987).

${ }^{26}$ K. Keem, H. Kim, G. T. Kim, J. S. Lee, B. Min, K. Cho, M. Y. Sung, and S. Kim, Appl. Phys. Lett. 84, 4376 (2004).

${ }^{27}$ J. Zhou, Y. Gu, Y. Hu, W. Mai, P. H. Yeh, G. Bao, A. K. Sood, D. L. Polla, and Z. L. Wang, Appl. Phys. Lett. 94, 191103 (2009).

${ }^{28}$ Y. Li, F. Della Valle, M. Simonnet, I. Yamada, and J. J. Delaunay, Appl. Phys. Lett. 94, 23110 (2009). 